\title{
Exploring the validity of allostatic load in pregnant women
}

\author{
Yang $\mathrm{Li}^{\mathrm{a}, *}$, Vanessa K. Dalton ${ }^{\mathrm{b}}$, Shawna J. Lee ${ }^{\mathrm{c}}$, Marie-Anne S. Rosemberg ${ }^{\mathrm{d}}$, Julia S. Seng ${ }^{\mathrm{d}}$ \\ a Sinclair School of Nursing, University of Missouri, Columbia, MO 65211, USA \\ b Department of Obstetrics and Gynecology Medical School, University of Michigan, Ann Arbor, MI, USA \\ ' School of Social Work, University of Michigan, Ann Arbor, MI, USA \\ d School of Nursing, University of Michigan, Ann Arbor, MI, USA
}

\section{A R T I C L E I N F O}

\section{Article history:}

Received 1 July 2019

Revised 21 December 2019

Accepted 27 December 2019

\section{Keywords:}

Allostatic load

Pregnancy

Validity

Sociodemographic stress

Chronic stress

\begin{abstract}
A B S T R A C T
Background: The theory of allostatic load has gained momentum in perinatal research to understand the biological pathways of the impact of maternal chronic stress on adverse perinatal outcomes. However, due to physiological changes of pregnancy, including large variations across gestation, the extent to which allostatic load measured in pregnancy is valid has not been queried in depth.
\end{abstract}

Objectives: This study aimed to assess the gestational patterns of selected individual allostatic load indicators and to explore whether a pregnancy allostatic load index score had face validity in relation to chronic sociodemographic stress.

Design: This is a secondary analysis using data from the 1999-2006 National Health and Nutrition Examination Survey.

Methods: A total of 1056 pregnant women were included for analyses. Using ten physiological indicators commonly included in an index of allostatic load, we described individual indicator and index score patterns across gestation and assessed differences in allostatic load index scores between women with and without sociodemographic stress.

Findings: The average allostatic load index score at any gestational month was not statistically significantly different from the average allostatic load index score in the non-pregnant sample. We also found the allostatic load index score remained steady across gestational month, despite very different gestational patterns of individual allostatic load indicators, as long as gestation-specific risk quartiles were used to calculate the allostatic load index score. Face validity was affirmed via higher allostatic load index scores in women with sociodemographic stress compared to those without such stress.

Conclusion: Our study suggests that measuring allostatic load in pregnancy is valid for reflecting women's true physiological functions and chronic stress, but gestational age should be considered when scoring the allostatic load index for women at different gestational age.

Implications for practice: As a valid measure of chronic stress, a pregnancy allostatic load index may contribute to research on health inequities and perinatal outcomes.

(C) 2019 Elsevier Ltd. All rights reserved.

\section{Introduction}

Chronic or repeated stress such as low socioeconomic status and child maltreatment has been demonstrated in relation to increased risk for morbidity and mortality (Felitti et al., 1998; Gilbert et al., 2009; Nandi et al., 2014). The long-term costs of chronic or repeated stress on biological stress response systems could explain how exposures to stress shape health outcomes. Bio-

\footnotetext{
* Corresponding author.

E-mail address: liy5@missouri.edu (Y. Li).
}

logical stress responses are activated to successfully adapt to stressors in the short term, but in the long term the prolonged activation of stress response systems results in dysregulations of physiological mediators from neuroendocrine, immune, cardiovascular, and metabolic body systems and thus eventually leads to diseases. The "wear and tear" effect on body systems induced by chronic stress is the basic assumption of the allostatic load (AL) theory.

AL refers to the cumulative physiological dysregulations of multiple body systems (e.g., neuroendocrine, immune, cardiovascular, and metabolic systems) responsive to chronic stress (McEwen and Stellar, 1993). In response to stressful environmental demands, the activity of the neuroendocrine system is acti- 
vated and primary mediators (i.e., cortisol, norepinephrine, and epinephrine) are released, causing a cascade of physiological effects on other body systems such as immune, metabolic, and cardiovascular systems. Repeated, prolonged, or inadequate stress responses cause systemic dysregulations of biomarkers from multiple systems and can eventually lead to diseases (McEwen, 2006). The theory of AL describes a process from stress to diseases and provides a framework for understanding the detrimental effects of repeated or chronic stress on poor health outcomes.

AL is commonly operationalized as a composite index (AL index; ALI) score that combines multiple biomarkers and anthropometric indicators from across several body systems. A higher ALI score indicates worse body system functioning. AL has been well documented in relation to both chronic stress and adverse health outcomes in non-pregnant populations (Beckie, 2012; Juster et al., 2010). In a systematic review of 11 studies using data from the National Health and Nutrition Examination Survey (NHANES) with slightly different combinations of AL individual indicators, ALI scores varied across sociodemographic factors (Beckie, 2012). AL levels increased with age from 20 to 60 years. Black people had higher AL levels than White people at all ages. Lower education and income levels were also associated with higher AL levels across all ethnic groups (Beckie, 2012). Some studies also suggested AL plays a mediating role in the link between stress and health outcomes (Crimmins et al., 2009; Seeman et al., 2004). Applying the AL theory to perinatal research, AL may serve as a potential contributor to adverse perinatal outcomes in women who experienced psychosocial and traumatic stress. The ALI score may be a new risk assessment method to detect the risk for adverse perinatal outcomes among pregnant women experiencing psychosocial and traumatic stress.

However, only a small number of studies have measured AL during pregnancy (Hux and Roberts, 2015; Morrison et al., 2013; Wallace and Harville, 2013). Pregnancy is a state that involves temporary alterations in physiology across multiple body systems to facilitate reproduction. These alterations have different patterns across gestation. For example, blood pressure declines until the second trimester and then rise in the third trimester, total cholesterol (TC) and triglycerides increase across gestation, and high-density lipoprotein (HDL) rises in the first and second trimester and then decreases in the third trimester (SomaPillay et al., 2016). In addition to the normal basal physiological alterations in pregnancy, physiological stress response systems that constitute AL are modified compared with the non-pregnant state (Blackburn, 2014). A review of 15 studies that used standardized laboratory stressors to test stress reactivity to pain and psychological stress during pregnancy found that physiological stress responses (i.e., blood pressure, heart rate, cortisol, epinephrine, norepinephrine) to exogenous challenges might be attenuated during pregnancy (de Weerth and Buitelaar, 2005). Thus, there is a question about the extent to which the great changes in physiology and physiological stress reactivity during pregnancy affect individual AL indicators from neuroendocrine, immune, cardiovascular, and metabolic systems in ways that reduce the validity of AL measurement. And therefore, it is unclear whether AL in pregnancy validly reflects women's true physiological status as well as whether it can be a valid indicator of chronic stress such as sociodemographic stress.

For this study we used NHANES data to address two aims: A) To examine the gestational curves of each individual AL indicator and of an ALI score and determine implications for scoring, and B) to test whether the ALI score has face validity in pregnancy by comparing women with and without sociodemographic stress in terms of ALI scores.

\section{Methods}

\section{Study design}

This is a secondary analysis using NHANES data. NHANES is a cross-sectional study designed to assess various aspects of health of adults and children in the United States, which has been conducted in a 2-year cycle since 1999 (CDC and NCHS, 2014). A complex, multistage probability sampling approach has been used to select a large nationally representative sample of approximately 5000 individuals annually from all 50 states and the District of Columbia. It consists of an interview administered in the home and a standardized physical examination conducted in a mobile examination center (MEC). The interview component of NHANES asks sociodemographic information, dietary behaviors, diseases, medical conditions, and other health-related questions. The examination component includes medical, dental, and physiological measurements, as well as numerous laboratory tests. To address the study aims, we used data from the 1999 to 2006 cycles of NHANES. The data collected after 2006 were not used because since 2007 pregnant women have not been oversampled. This secondary analysis of de-identified data was exempt from ethics review by the Institutional Review Board (IRB).

\section{Participants}

Pregnant women were included in the study. Pregnancy status at the time of the MEC examination was reported for females 8-59 years of age and confirmed through urine pregnancy test. If the urine test was positive, the status is coded as pregnant at examination. A total of 1256 women were pregnant at the examination, but 198 pregnant women (15.8\%) did not report their gestational month at the exam and two women (0.2\%) reported they were at gestational month 10 . These 200 women were excluded. Thus, a total of 1056 pregnant women were included for analyses.

\section{Variables and data sources}

The 10 physiological indicators used in the ALI were C-reactive protein (CRP) from the immune system; systolic blood pressure (SBP), diastolic blood pressure (DBP), and pulse from the cardiovascular system; and body mass index (BMI), TC, HDL, glycohemoglobin, glucose, and triglycerides from the metabolic system. These 10 physiological indicators have been frequently used in previous AL studies (Juster et al., 2010). The study did not include other physiological indicators from the NHANES database because there is a large amount of missing data or some of those indicators were collected only in subsamples. Standard examination and laboratory procedures were described in the NHANES Examination and Laboratory Protocols (CDC and NCHS, 2015).

To measure gestational month, we used the survey question - "What month of pregnancy are you in?" from the Reproductive Health Questionnaire. Sociodemographic information was also obtained from the household and MEC interviews. These items included age at the time of the MEC exam, race, poverty-income ratio, education levels, and marital status. In this study, age was dichotomized into two categories: 15-34 and 3544 years. Race was recoded as non-Hispanic White and other races (i.e., Mexican American, other Hispanic, non-Hispanic Black, and other race including multi-racial). Poverty-income ratio is an index computed by dividing family income by the Department of Health and Human Services (DHHS) poverty line, specific to family size, as well as the appropriate year and state. It 

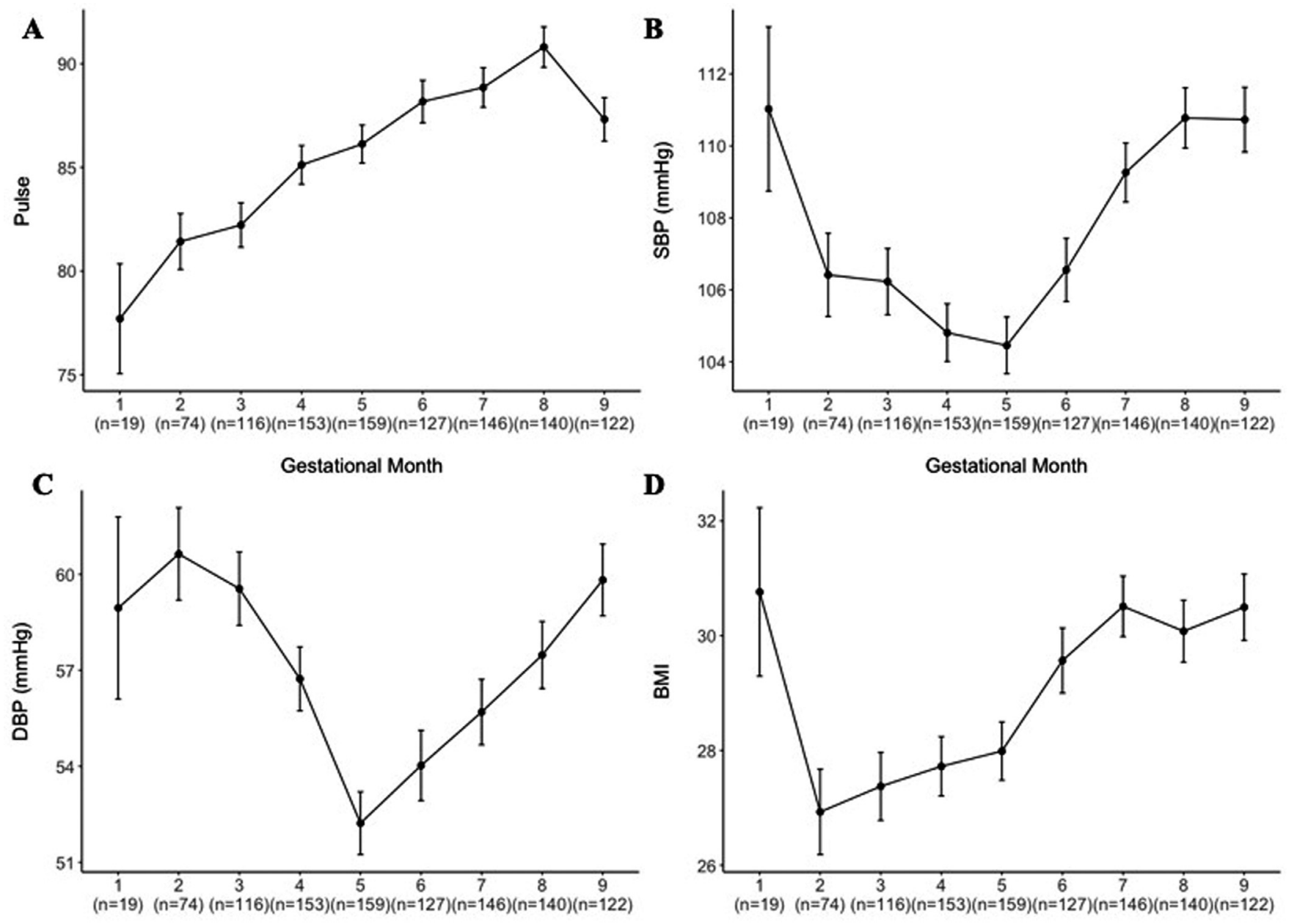

Gestational Month

Gestational Month
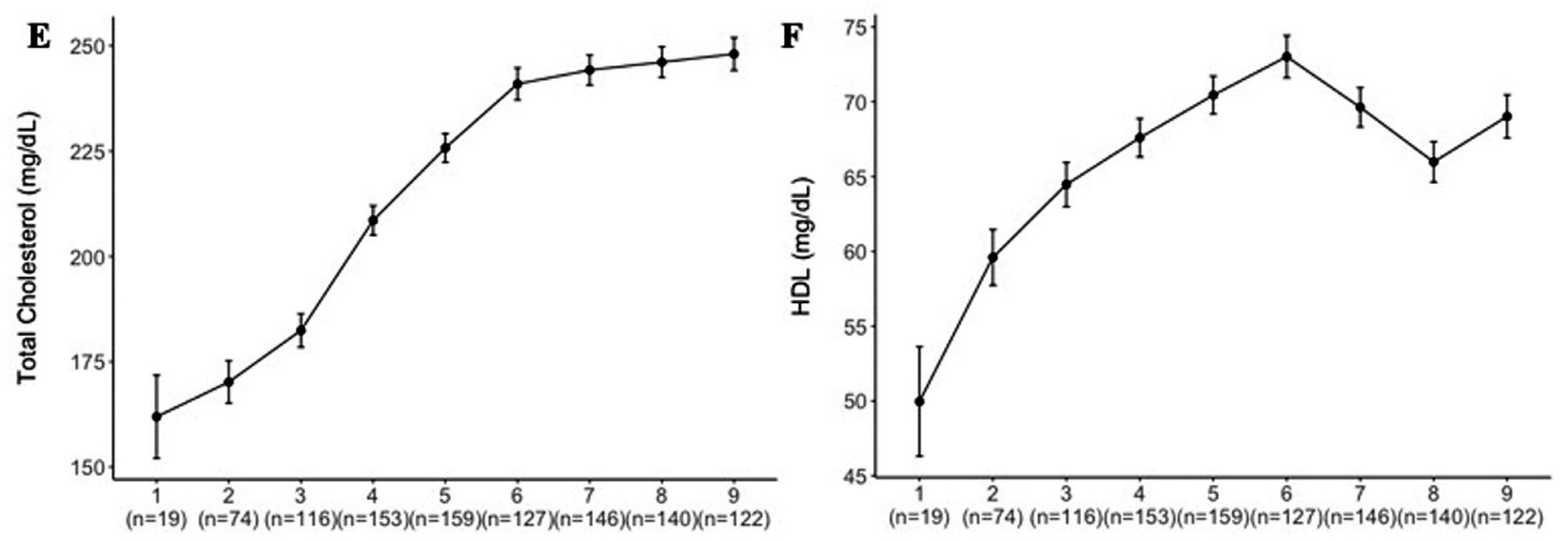

Gestational Month

\section{Gestational Month}

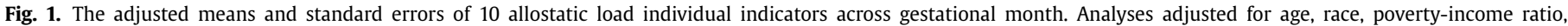
education level, and marital status.

ranges from 0 to 5 , with a lower number indicating more severe poverty. In this study, it was coded into two categories: low and middle poverty-income ratio (0-4) and high ratio (4.01-5). Education level was recoded as high school or less and more than high school. Marital status was coded as married/living with a partner and widowed, divorced, separated, or never married.
We created a proxy variable for sociodemographic stress by adding the number of high-stress categories the woman belonged to: being pregnant at the age of 35-44 years, other races except non-Hispanic White, having high school or less education, having the more severe poverty-income ratio (0-4), and being widowed, divorced, separated, or never married. The index was then 


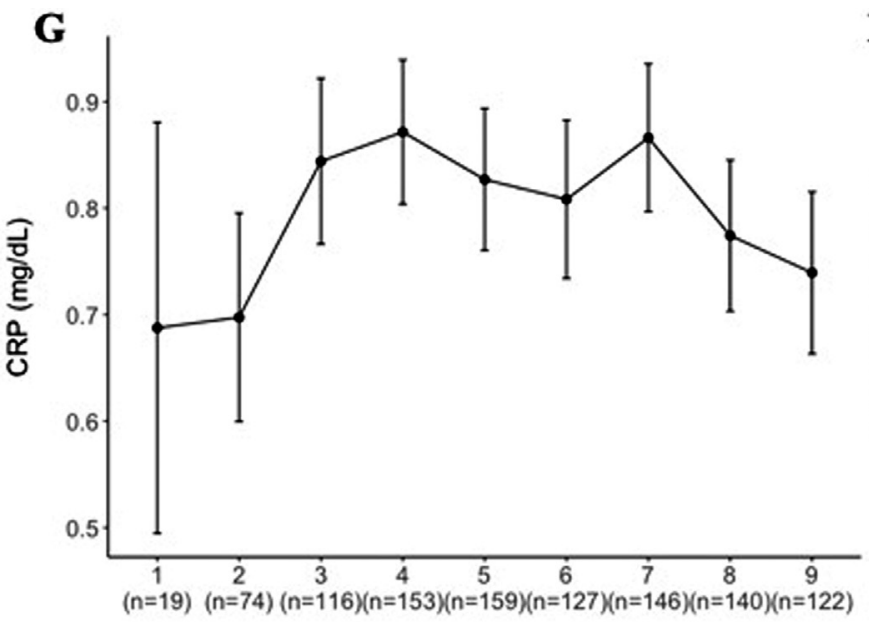

Gestational Month

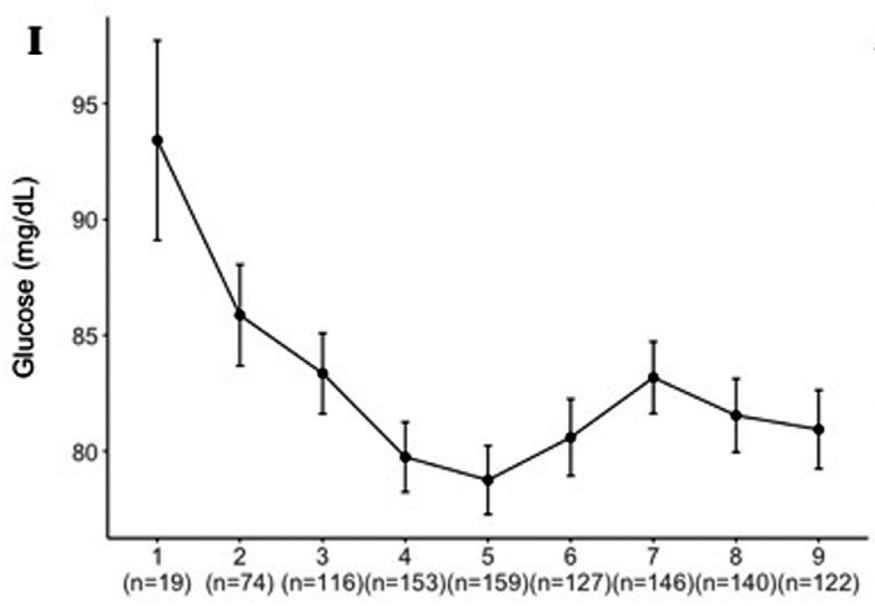

Gestational Month

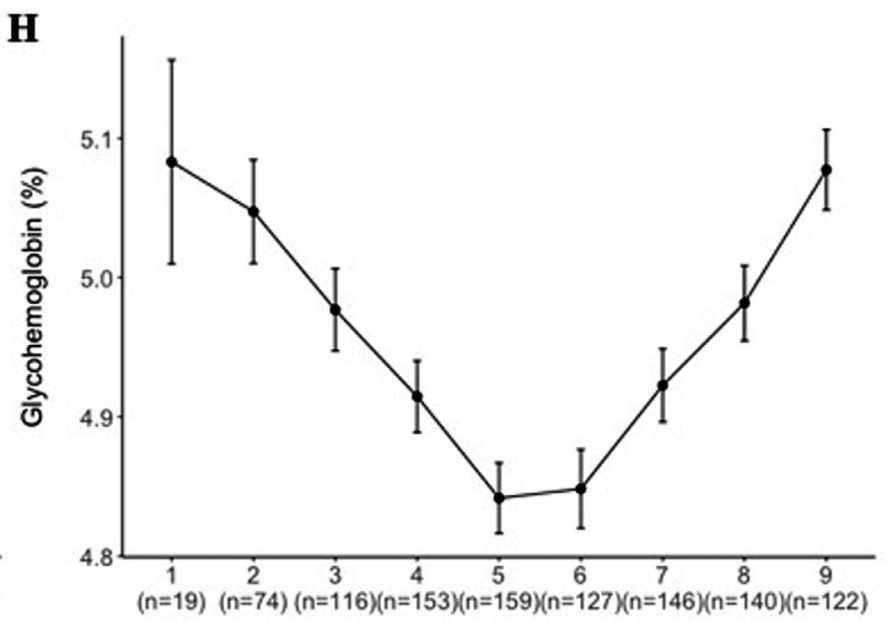

Gestational Month

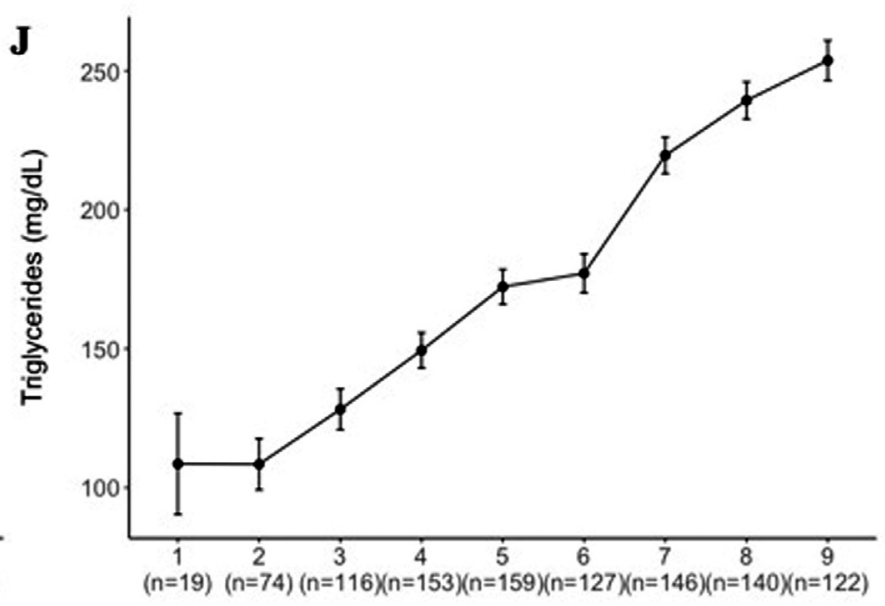

Gestational Month

Fig. 1. Continued

dichotomized, with the value of 0 indicating no sociodemographic stress and the range of $1-5$ indicating any stress.

\section{AL scoring}

The count-based method was used to score ALI (Li et al., 2019). Using this method, the ALI score is the number of physiological indicators on which participants scored in the highest risk quartile, yielding a possible score range of 0 to 10 .

We expected the levels of each physiological indicator to change across pregnancy. In this study, pregnant women were at different gestational month. Using the risk quartile for each physiological indicator, based on the whole pregnant sample distribution, would introduce error. Women with the most relatively elevated levels of physiological indicators at certain gestational months may be classified in the high-risk quartile, when in fact the level may be more closely associated with gestational months than stress physiology. Thus, the ALI calculations by the count-based method must account for gestational month. To do this, we divided the whole sample into 9 gestational-month-specific subsamples. Then we scored ALI within each gestational-age specific subsample. That is, we calculated the risk quartile for each physiological indicator based on the distribution within each of the 9 subsamples in which participants were at the same gestational month. The gestational-month-specific ALI was also dichotomized, with values at or above the 75th percentile considered high risk for poor health and scored as " 1 " while values below 75 th percentile scored as " 0 ".

In addition to the gestational-month-specific ALI score, we also calculated an ALI score without taking gestational month into account (Non-gestational-month-specific ALI score). That is, the cutoff points for each indicator were based on the distribution of each physiological indicator in the whole pregnant sample instead of each gestational-age specific subsample.

\section{Statistical analysis}

"Refused" or "don't know" responses were recoded as missing values. We used the multiple imputation (MI) method (Rubin, 2004) to impute all missing data. Based on the recommendation of Yuan (2010) with $13.9 \%$ of data missing, we created 10 imputed datasets (Yuan, 2010).

Each individual AL indicator was plotted for each gestationalmonth group to describe the gestational patterns of each individual indicator. Age, race, poverty-income ratio, education levels, and marital status were adjusted for in order to eliminate the heterogeneity of these gestational-month groups. Linear regression models were conducted to examine the effect of gestational month on each physiological indicator level. Each indicator was included respectively as the outcome variable and age, race, poverty-income ratio, education levels, and marital status were adjusted for in the regression models. Based on the plots, gestational month was in- 
Table 1

Summary of 10 physiological indicators in terms of their gestational patterns.

\begin{tabular}{|c|c|c|c|}
\hline Indicators & Patterns observed & $\begin{array}{l}\text { Statistically significant changes across } \\
\text { gestation by month or trimester? }\end{array}$ & $\begin{array}{l}\text { Consistent with known pregnancy } \\
\text { physiology? }\end{array}$ \\
\hline Pulse & $\begin{array}{l}\text { Near linear. Pulse increased with the } \\
\text { progression of pregnancy but had a } \\
\text { slight decrease at late gestation. }\end{array}$ & $\begin{array}{l}\text { Yes. Gestational month was } \\
\text { significantly correlated with pulse } \\
(\beta=0.22, P<0.001) \text {. }\end{array}$ & $\begin{array}{l}\text { Yes. The early increase in ventricular } \\
\text { wall muscle mass and end-diastolic } \\
\text { volume contribute to an increase in } \\
\text { stroke volume and heart rate in } \\
\text { pregnancy (Soma-Pillay et al., 2016). }\end{array}$ \\
\hline SBP & $\begin{array}{l}\text { Inverted bell curve. There was a } \\
\text { decrease in SBP from early gestation to } \\
\text { mid-gestation and then SBP increased } \\
\text { during late gestation. }\end{array}$ & $\begin{array}{l}\text { Yes. SBP at the first trimester was not } \\
\text { significantly higher than the second } \\
\text { trimester }(\beta=0.14, P=0.071) \text {, but SBP } \\
\text { in the third trimester was significantly } \\
\text { higher than the second trimester } \\
(\beta=0.16, P<0.001) \text {. }\end{array}$ & $\begin{array}{l}\text { Yes. The smooth muscle relaxation } \\
\text { and overall vasodilation caused by } \\
\text { elevated progesterone contribute to } \\
\text { the decreased SBP in the first and } \\
\text { second trimesters. The highly } \\
\text { increased plasma volume in the third } \\
\text { trimester causes an increase in SBP } \\
\text { (Soma-Pillay et al., 2016). }\end{array}$ \\
\hline DBP & $\begin{array}{l}\text { Inverted bell curve. DBP decreased until } \\
\text { the middle of gestation and then } \\
\text { increased to the late gestation. }\end{array}$ & $\begin{array}{l}\text { Yes. DBP at the first and third } \\
\text { trimester were significantly higher } \\
\text { than the second trimester }(\beta=0.39 \text {, } \\
P<0.001 ; \beta=0.08, P<0.001) \text {. }\end{array}$ & $\begin{array}{l}\text { Yes. The explanation for the pattern of } \\
\text { DBP is the same as SBP } \\
\text { (Soma-Pillay et al., 2016). }\end{array}$ \\
\hline BMI & $\begin{array}{l}\text { Inverted bell curve. BMI decreased in } \\
\text { the first month, increased to gestational } \\
\text { month } 7 \text {, and then remained steady for } \\
\text { the last } 2 \text { months of gestation. }\end{array}$ & $\begin{array}{l}\text { Yes. BMI at the first and second } \\
\text { trimester were significantly lower } \\
\text { than the third trimester }(\beta=-0.39 \text {, } \\
P<0.001 ; \beta=-0.10, P<0.001) \text {. }\end{array}$ & $\begin{array}{l}\text { Yes. Pregnant women lose weight at } \\
\text { early gestation which may be due to } \\
\text { nausea and vomiting. After the } \\
\text { symptoms reduce, their weight } \\
\text { increases. }\end{array}$ \\
\hline TC & $\begin{array}{l}\text { Near linear. There was a steady increase } \\
\text { in TC as pregnancy progresses. }\end{array}$ & $\begin{array}{l}\text { Yes. Gestational month was } \\
\text { significantly correlated with TC } \\
(\beta=0.49, P<0.001) .\end{array}$ & $\begin{array}{l}\text { Yes. The increase in TC levels is } \\
\text { mainly due to increased synthesis by } \\
\text { the liver and decreased lipoprotein } \\
\text { lipase activity, resulting in decreased } \\
\text { catabolism of adipose tissue. Changes } \\
\text { in lipid metabolism accommodate the } \\
\text { needs of the developing fetus. } \\
\text { Increased TC levels provide for the } \\
\text { mother's energy needs while glucose } \\
\text { is spared for the fetus } \\
\text { (Soma-Pillay et al., 2016). }\end{array}$ \\
\hline HDL & $\begin{array}{l}\text { Bell curve. HDL increased in the first } \\
\text { half of pregnancy and then fall in the } \\
\text { third trimester but the level was still }\end{array}$ & $\begin{array}{l}\text { Yes. HDL levels at the first trimester } \\
\text { were significantly lower than the } \\
\text { second trimester }(\beta=-0.47, P<0.001) \text {, }\end{array}$ & $\begin{array}{l}\text { Yes. The explanation for the pattern of } \\
\text { HDL is the same as TC } \\
\text { (Soma-Pillay et al., 2016). }\end{array}$ \\
\hline
\end{tabular}

second trimester $(\beta=-0.47, P<0.001)$

higher than early pregnancy.

but the levels at the third trimester

were not significantly lower than

those of the second trimester

$(\beta=-0.04, P=0.086)$.

No. CRP levels during the first and

third trimester were not significantly

CRP levels fluctuated

levels reached at gestational month 4

lower than the second trimester

$(\beta=-0.06, P=0.430 ; \beta=-0.02$,

and 7.

$P=0.503)$.

Glycohemoglobin

Inverted bell curve. Glycohemoglobin fell in the half of pregnancy and then rose to the late pregnancy.

Glucose

Inverted bell curve. Glucose decreased in the middle of gestation and then increased to the late gestation.
Yes. Glycohemoglobin levels at the first and third trimester were significantly higher than the second trimester $(\beta=0.38, P<0.001 ; \beta=0.11$, $P<0.001)$.

Yes. Glucose levels at the first trimester were significantly higher than the second trimester $(\beta=0.27$, $P==0.005$ ), but the levels at the third trimester were not significantly higher than the second trimester $(\beta=0.04, P=0.118)$.

Yes. Gestational month was significantly correlated with triglycerides levels $(\beta=0.50, P<0.001)$.
Consistent with known pregnancy

Yes. The early increase in ventricula muscle mass and end-diastolic stroke volume and heart rate in pregnancy (Soma-Pillay et al., 2016). Yes. The smooth muscle relaxation the decreased SBP in the first and trimesters. The highly (Soma-Pillay et al., 2016).

Yes. The explanation for the pattern of DBP is the same as SBP

Yes. Pregnant women lose weight at early gestation which may be due to mainly due to increased synthesis by the liver and decreased lipoprotein ism of adipose tissue. Changes Increased TC levels provide for the is spared for the fetus (Soma-Pillay et al., 2016).

Partially consistent. CRP is known to be slightly elevated during pregnancy. This is because the maternal inflammatory reaction to the pregnancy. But there is no consistent change in CRP levels with gestational age (von Versen-Hoeynck et al., 2009; Watts et al., 1991).

Yes. The pattern is the result of increased insulin secretion and increased insulin sensitivity in early pregnancy, followed by progressive insulin resistance in the third trimester (Soma-Pillay et al., 2016). Yes. The explanation for the pattern of glucose is the same as glycohemoglobin (Soma-Pillay et al., 2016).

Yes. The explanation for the pattern of triglycerides is the same as TC (Soma-Pillay et al., 2016).

$\mathrm{SBP}=$ Systolic blood pressure; DBP = Diastolic blood pressure; BMI = Body mass index; TC = Total cholesterol; HDL $=$ High-density lipoprotein; $\mathrm{CRP}=C$-reactive protein .

cluded as an explanatory variable when the relationship between gestational month and the indicator was linear, while trimester was included as the explanatory variable when the relationship was not linear.
The average gestational-month-specific ALI score was plotted for each gestational-month group to describe changes in ALI scores across different gestational month. To eliminate the heterogeneity of these gestational-month groups, we adjusted for age, race, poverty-income ratio, education level, and marital status. Means 
and standard deviations were used to describe gestational-monthspecific ALI scores in terms of different sociodemographic factors and the dichotomized sociodemographic stress index.

In order to compare the average gestational-month-specific ALI scores with the average ALI score in the non-pregnant sample (women with reproductive ages of 15-49), we computed the average ALI score by the count-based method from the NHANES 2001-2006 database and added to the graph of the pregnancy ALI scores (Li et al., 2019). As comparison, we also plotted the average non-gestational-month-specific ALI score for each gestationalmonth group with the average ALI in the non-pregnant sample added to the plot.

Three binary logistic regression models were conducted to examine the associations between gestational age, sociodemographic stress, and AL, with the dichotomized gestational-month specific ALI score as the dependent variable. Although we computed the gestational-month-specific ALI score, we still included gestational month in the models to control for other gestational factors such as pregnancy stress. In the first model, only gestational month was included as the explanatory variable. In the second model, gestational month, age, race, poverty-income ratio, education levels, and marital status were included as the explanatory variables. In the third model, gestational month and the dichotomized sociodemographic stress index were included as the explanatory variables. All statistical analyses were performed using R Software Version 3.4.2 (R Core Team, 2017).

\section{Results}

\section{The gestational patterns of each individual indicator}

As seen in Fig. 1, pulse, TC, and triglycerides increased with the progression of pregnancy. SBP, DBP, glycohemoglobin, and glucose decreased until the middle of gestation and then increased to the late gestation, with the lowest level during the second trimester. BMI decreased dramatically from gestational month 1 to month 2, increased gradually from month 2 to month 7, and then remained stable for the last two months of pregnancy. Regarding HDL and CRP, there was an increase from early gestation to the middle of gestation and then a decrease in late gestation. Except for CRP, there were significant differences in each individual AL indicator levels across different gestational month or trimesters (Table 1).

The descriptive statistics of gestational-month-specific ALI scores in terms of sociodemographic factors

As seen in Table 2, pregnant women aged 35-44 years had significantly higher ALI scores than those aged $15-34$ years $(t=-2.55$, $p=0.011$ ). Women with poverty-income ratio no more than 4 had significantly higher ALI scores compared to those with povertyincome ratio more than $4(t=2.64, p=0.008)$. There were no statistically significant differences in ALI scores between nonHispanic White and other races, women with high school or less education level and those with more than high school educational level, and women who were married or living with partner and those who were widowed, divorced, separated, or never married $(p>0.05)$. Compared to those without sociodemographic stress, pregnant women with any sociodemographic stress had significantly higher ALI scores $(t=-2.68, p=0.008)$.

\section{The gestational curves of ALI scores}

As seen in Fig. 2a, the non-gestational-month-specific ALI scores changed significantly across gestational months. Except for gestational month 2 and 6, the ALI scores at each gestational month were different from the average ALI score in the non-pregnant

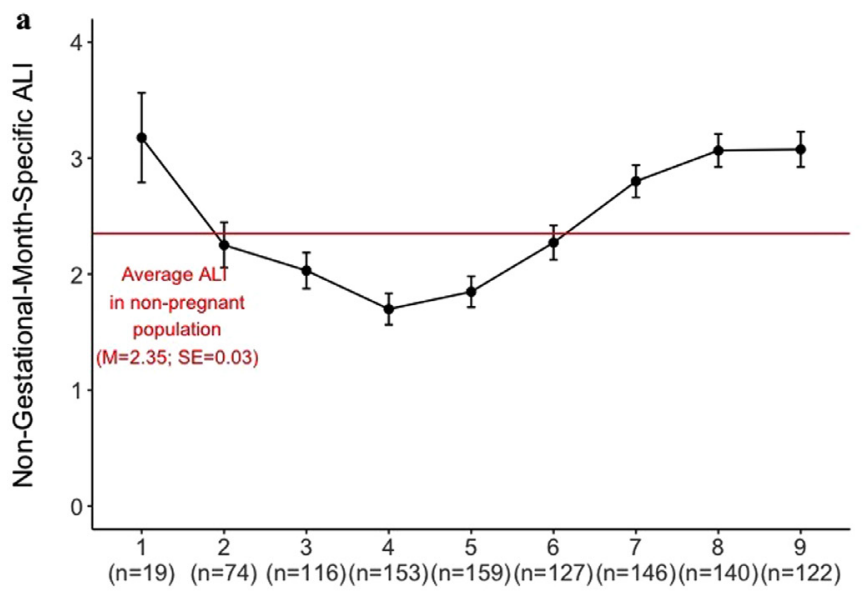

Gestational Month

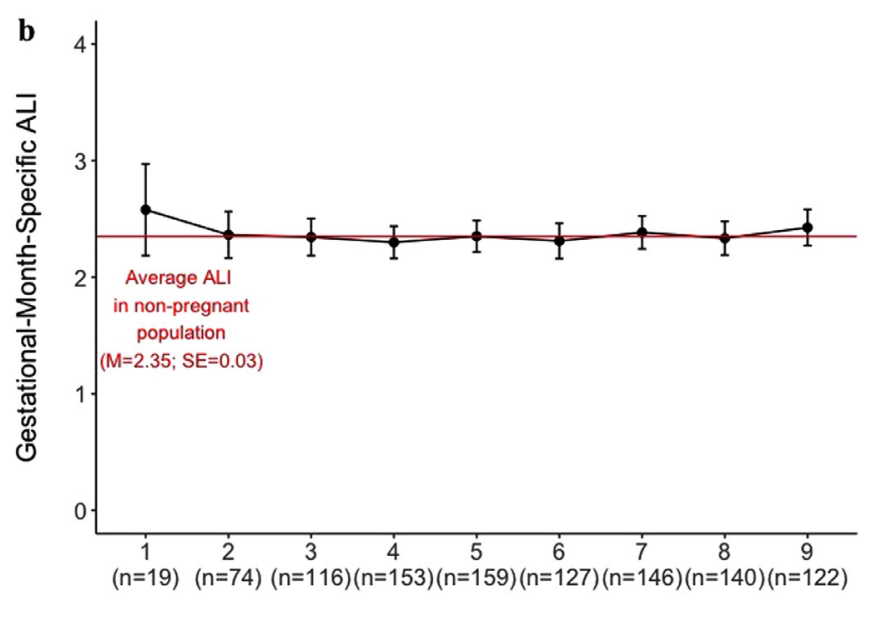

Gestational Month

Fig. 2. (a) The adjusted mean and standard error of non-gestational-month-specific allostatic load index scores across gestational month. The average allostatic load index score in the non-pregnant population (women with reproductive ages of 15-49) from the NHANES 2001-2006 database was also shown for comparison. Analyses adjusted for age, race, poverty-income ratio, education level, and marital status. (b) The adjusted mean and standard error of gestational-month-specific allostatic load index scores across gestational month. The average allostatic load index score in the non-pregnant population (women with reproductive ages of 15-49) from the NHANES 2001-2006 database was also shown for comparison. Analyses adjusted for age, race, poverty-income ratio, education level, and marital status.

population (women of reproductive age) from the NHANES 20012006 database $(M=2.35, \mathrm{SE}=0.03, N=4319)$. However, after we took gestational month into account when scoring ALI scores, the gestational-month-specific ALI scores remained steady across gestational month and the score at each gestational month was around the score in the non-pregnant sample (Fig. 2b). The average ALI score in the non-pregnant population was within one standard error above and below the average pregnancy ALI score at each gestational month. The gestational-month-specific ALI scores were not statistically significantly different from the average ALI score in the non-pregnant population.

The associations between $A L$, gestational month, and sociodemographic stress

As seen in Table 3, the binary logistic regression model verified that gestational month was not significantly associated with the ALI scores, which had been scored with gestational-month specific risk quartiles $(p>0.05)$. The association remained non- 
Table 2

Descriptive statistics: Association between allostatic load index scores and sociodemographic factors $(N=1056)$.

\begin{tabular}{lllll}
\hline & \multicolumn{2}{l}{ ALI scores } & & \\
\cline { 2 - 5 } & $n$ & $M \pm S D$ & $t$ value & $p$ \\
\hline Age & & & -2.55 & .011 \\
$\quad 15-34$ & 935 & $2.31 \pm 1.69$ & & \\
$\quad 15-44$ & & $2.73 \pm 1.83$ & & \\
$\quad$ Race & 482 & $2.35 \pm 1.68$ & & .025 \\
$\quad$ Non-Hispanic White & 574 & $2.36 \pm 1.73$ & & \\
$\quad$ Other races & & & 2.64 & .008 \\
$\quad \begin{array}{l}\text { Poverty-income ratio } \\
\quad 4\end{array}$ & 793 & $2.44 \pm 1.73$ & & \\
$\quad>4$ & 263 & $2.12 \pm 1.62$ & & \\
$\quad \begin{array}{l}\text { Education level } \\
\quad \text { High school or less }\end{array}$ & 549 & $2.40 \pm 1.67$ & & .342 \\
$\quad$ More than high school & 507 & $2.30 \pm 1.75$ & & \\
$\quad$ Marital status & & & 0.49 & .626 \\
$\quad$ Married/living with partner & 803 & $2.37 \pm 1.71$ & & \\
$\quad$ Widowed/divorced/separated/never married & 253 & $2.31 \pm 1.70$ & & \\
$\quad$ Sociodemographic stress & & & -2.68 & .008 \\
$\quad$ No & 135 & $1.99 \pm 1.70$ & & \\
$\quad$ Any & 921 & $2.41 \pm 1.70$ & & \\
\hline
\end{tabular}

$\mathrm{ALI}=$ Allostatic load index.

a Sociodemographic stress is the sum of disadvantaged categories: older age (35-44 years), racial/ethnic groups besides non-Hispanic White, poverty-income ratio less than or equal to

4 , high school or less education level, or widowed, divorced, separated, or never married.

Table 3

Associations between allostatic load, gestational month, and sociodemographic stress.

\begin{tabular}{|c|c|c|c|c|c|c|c|c|c|}
\hline & \multicolumn{3}{|c|}{ Model 1} & \multicolumn{3}{|c|}{ Model 2} & \multicolumn{3}{|c|}{ Model 3} \\
\hline & OR & $95 \% \mathrm{CI}$ & $P$ & OR & $95 \% \mathrm{CI}$ & $P$ & OR & $95 \% \mathrm{CI}$ & $P$ \\
\hline Gestational month & 1.05 & {$[0.99,1.11]$} & .122 & 1.05 & {$[0.99,1.11]$} & .115 & 1.05 & {$[0.99,1.11]$} & .119 \\
\hline Aged $35-44$ years $^{\mathrm{a}}$ & & & & 1.46 & {$[0.98,2.15]$} & .081 & & & \\
\hline Other races except Non-Hispanic White ${ }^{b}$ & & & & 0.92 & {$[0.69,1.21]$} & .547 & & & \\
\hline Low and middle poverty-income ratio ${ }^{c}$ & & & & 1.30 & {$[0.93,1.82]$} & .183 & & & \\
\hline High school or less ${ }^{\mathrm{d}}$ & & & & 1.08 & {$[0.80,1.46]$} & .609 & & & \\
\hline Divorced/Separated/Widowed ${ }^{e}$ & & & & 0.97 & {$[0.70,1.33]$} & .592 & & & \\
\hline Any sociodemographic stress ${ }^{\mathrm{f}}$ & & & & & & & 1.56 & {$[1.06,2.37]$} & .043 \\
\hline
\end{tabular}

a Aged 15-34 years was the reference.

b Non-Hispanic White was the reference.

c High poverty-income ratio (>4) was the reference.

d More than high school education level was the reference.

e Married/living with partner was the reference.

${ }^{f}$ No sociodemographic stress (the sociodemographic stress index score is 0 ) was the reference.

significant after including all 5 individual sociodemographic factors (age, race, poverty-income ratio, education levels, and marital status) or the sociodemographic stress in the model $(p>0.05)$. All 5 sociodemographic factors were also not significantly associated with ALI scores $(p>0.05)$. But women with any sociodemographic stress had significantly higher ALI scores than those without sociodemographic stress $(\mathrm{OR}=1.56,95 \% \mathrm{Cl}=[1.06,2.37])$. The gestational curves of ALI scores by no and any sociodemographic stress are shown in Fig. 3.

\section{Discussion}

This study described the gestational patterns of each individual AL indicator and ALI scores as well as assessed the validity of measuring AL in pregnancy. We found strong patterns of differences in levels of each individual AL indicator (SBP, DBP, pulse, BMI, TC, HDL, glycohemoglobin, glucose, and triglycerides) at different gestational months except for CRP. When calculated using gestationalmonth specific risk quartiles, ALI score remained steady across gestation, and was not statistically different from the average score in the non-pregnant NHANES sample (women with reproductive ages of 15-49). We also found higher sociodemographic stress in rela- tion to elevated ALI scores, suggesting face validity of the ALI score in relation to chronic stress measured in pregnancy.

Except for CRP, each individual AL indicator changed significantly across gestational month. The changing pattern of each indicator across gestation was consistent with known pregnancy physiology. The finding suggests that gestational age should be taken into account when scoring AL in pregnant women. Since physiological indicators included in the ALI change with pregnancy, population-based pregnancy-specific cut-off points need to be determined for each individual AL indicator at each gestational month. Given that the NHANES has a nationally representative sample with data collected with standardized procedures and protocols, future research could identify the cut-off points of each individual AL indicator for each gestational month using the NHANES data.

We found that the non-gestational-month-specific ALI score changed across gestational months. But after we considered the influence of gestational age when scoring ALI, the gestational-monthspecific ALI score remained steady across gestation and the score at each gestational month was not different from the scores in the non-pregnant population. This study finding may indicate that measuring AL at any time in pregnancy would reflect women's true physiological functions. We found no association between age, 


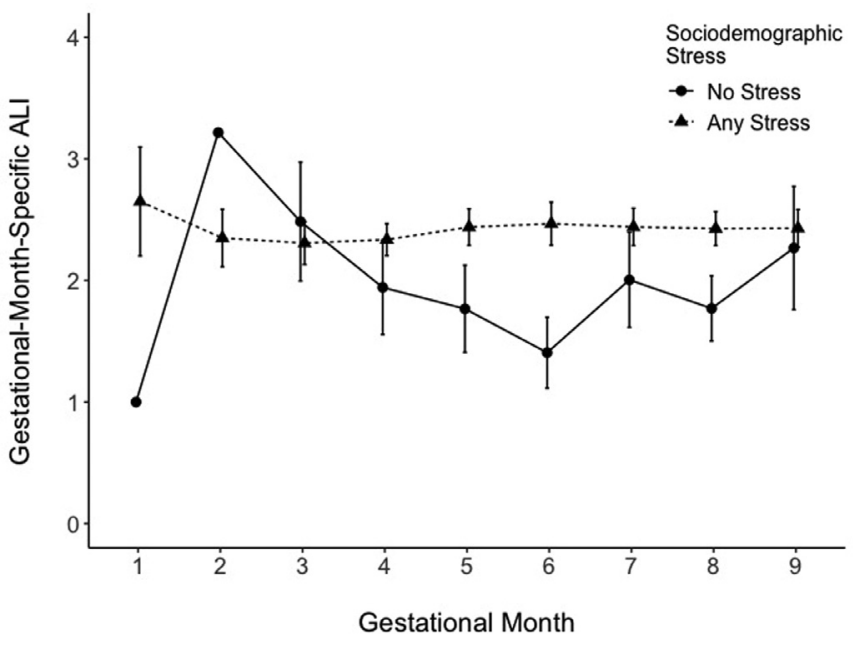

Fig. 3. The gestational-month-specific allostatic load index scores across gestational month in terms of sociodemographic stress. There is only one woman at Gestational Month 1 without sociodemographic stress.

race, poverty-income ratio, education level, and marital status with gestational-month-specific ALI scores. But women with any sociodemographic stress had higher ALI scores compared to those without sociodemographic stress. These findings may suggest that cumulative sociodemographic stress is a more significant predictor than individual sociodemographic factors for predicting elevated pregnancy AL levels. It may also indicate that measuring $A L$ in pregnancy could be a valid measure of chronic stress.

Few studies have examined AL in pregnant women. In these studies AL was measured $\mathrm{AL}$ at one time point in early or late pregnancy or at any time during pregnancy (Hux and Roberts, 2015; Morrison et al., 2013; Wallace and Harville, 2013). Only one study assessed the validity of measuring AL during pregnancy, using the same NHANES 1999-2006 data with pregnant women at different gestation months (Morrison et al., 2013). But the study failed to find validity in measuring AL during pregnancy. This was probably because their approach to scoring ALI did not account for changes in individual AL indicators across gestation. Morrison et al. (2013) also suggested that measuring AL in pregnancy might reflect women's exposure to chronic stress if pregnancy physiological changes were taken into account.

We found the biggest differences in ALI scores at gestational month 6 between women with and without sociodemographic stress. This finding suggests that the greatest variance in AL levels that can be explained by chronic stress may be in mid-pregnancy. This further indicates that the 6 month routine visit, might be the optimal time to collect individual AL indicators if the goal is to measure AL only once during pregnancy.

There are a few limitations in this study. First, given the crosssectional study design of the NHANES, pregnant women were at different gestational months and the ALI score was calculated in each gestational month group. We plotted the average ALI score of each gestational month group instead of the average score of the same sample across gestation to describe the gestational patterns of ALI scores. But to eliminate the heterogeneity of these gestational-month groups, we adjusted for age, race, povertyincome ratio, education levels, and marital status when plotting the gestational curve of the ALI score. Second, gestational month instead of gestational week was asked in the NHANES study, which may slightly influence our descriptions about the changing patterns of the ALI scores and each physiological indicator across gestation. Additionally, there was only a very small number of pregnant women at gestational month 1 and women at the very early stage of pregnancy may not know their exact gestational age, introducing a potential source of error in the first point on the gestational curve. For instance, although the increase of BMI from gestational month 2 to late gestation appears to be consistent with known pregnancy physiology, BMI was highest at gestational month 1, which seems unlikely to represent the effect of gestation on BMI. Also, there was only one woman at gestational month 1 without sociodemographic stress. Low education attainment is a factor in the sociodemographic stress index; thus potentially low health literacy about pregnancy may have result in women stating they are in month 1 erroneously. This source of error is unlikely to occur in clinical use, when gestational age would be determined by parameters other than self report. Lastly, data on biomarkers from the neuroendocrine system (i.e., cortisol, epinephrine, norepinephrine), which are direct primary mediators in response to stress, are lacking in the NHANES database. The ALI was constructed without biomarkers from the neuroendocrine system. Given the limitations of the study, a longitudinal study with pregnant women is needed to measure a full complement of individual AL indicators at multiple time points across gestation to replicate our analyses.

Despite these limitations, the study has strengths as well. The NHANES database includes numerous biomarker and anthropometric measures from the body systems usually measured in AL, which provides adequate individual AL indicators for our study to create a pregnancy ALI. Additionally, the NHANES data are collected with standardized procedures and protocols. This assure that the data for analyses are of high reliability and validity.

This study may also have some implications for further research. The study addressed an important methodological issue regarding measuring AL in pregnancy. For future studies that recruit pregnant women at different gestational ages, gestational age should be taken into account when scoring ALI. The study suggested that measuring AL in pregnancy could reflect women's true $\mathrm{AL}$ in relation to chronic stress, which contributes to applying AL to perinatal research on exploring AL as a potential biological mechanisms or pathways for the impact of maternal chronic stress on adverse perinatal outcomes. In addition, our study suggested that late second trimester is the optimal time of collecting individual AL indicators in order to detect the greatest variance in AL levels explained by chronic stress. Thus, future perinatal studies with limited study funding can select one time point to measure $\mathrm{AL}$; this could align with clinical routine laboratory assessments generally conducted in the 24-28 week period.

This study is an additional step toward verifying the validity of applying AL to perinatal outcomes research. Future investigations of the ALI score as a new risk assessment method may contribute to developing a comprehensive assessment of risk for adverse perinatal outcomes. The ALI score may be added to existing risk scoring systems and technical assessments to identify the majority of pregnant women subsequently having an adverse perinatal outcome. For pregnant women whose ALI score was particularly high, stress mitigation interventions in particular might prove valuable.

\section{Conclusion}

In summary, measuring $\mathrm{AL}$ in pregnancy is valid for reflecting women's true physiological functions and chronic stress, but gestational age needs to be taken into account when scoring AL. As a valid measure of chronic stress, pregnancy AL may prove to be a significant contributor to predicting adverse perinatal outcomes. Future studies measuring multi-system individual AL indicators at multiple time points during pregnancy and following up women's perinatal outcomes are needed to establish predictive validity. Given the toll of prolonged and severe stress and high AL on health across the lifespan, applying the theory of AL to perinatal 
health may contribute to quantification of psychosocial stress and advocacy for multiple eco-social levels of stress remediation for the most stress-exposed and thus vulnerable pregnant women.

\section{Ethical approval}

This is a secondary analysis of de-identified data. It was exempt from IRB review.

\section{Funding sources}

This research did not receive any specific grant from funding agencies in the public, commercial, or not-for-profit sectors.

\section{Declaration of Competing Interest}

Dr. Vanessa K. Dalton reports personal fees from Bayer, other from The Medical Letter, grants from Bill and Melinda Gates Foundation, grants from Blue Cross Blue Shield of Michigan Foundation (BCBSF), grants from NIH, grants from AHRQ grants from ECOG-ACRIN Medical Research Foundation, grants from Laura and John Arnold Foundation, grants from Susan Thomas Buffett Foundation, grants from Society of Family Planning, grants from Harvey I. Neiman Health Policy Institute, grants from University of Michigan, outside the submitted work. All the other authors declared no actual or potential conflict of interest.

\section{CRediT authorship contribution statement}

Yang Li: Conceptualization, Data curation, Formal analysis, Methodology, Project administration, Writing - original draft. Vanessa K. Dalton: Conceptualization, Methodology, Writing - review \& editing. Shawna J. Lee: Conceptualization, Writing - review \& editing. Marie-Anne S. Rosemberg: Conceptualization, Methodology, Writing - review \& editing. Julia S. Seng: Conceptualization, Methodology, Supervision, Writing - review \& editing.

\section{Acknowledgements}

The authors would like to thank the University of Michigan Consulting for Statistics, Computing and Analytics Research (CSCAR) consultants for their support on the statistical analyses of the study.

\section{References}

Beckie, T.M., 2012. A systematic review of allostatic load, health, and health disparities. Biol. Res. Nurs. 14 (4), 311-346.

Blackburn, S., 2014. Maternal, Fetal, \& Neonatal Physiology. Elsevier Health Sciences.
Centers for Disease Control and Prevention, National Center for Health Statistics. 2014. National health and nutrition examination survey overview. https://www. cdc.gov/nchs/data/nhanes/nhanes_13_14/NHANES_Overview_Brochure.pdf.

Centers for Disease Control and Prevention, National Center for Health Statistics. National Health and Nutrition Examination and Laboratory Protocols [19992006]. http://www.cdc.gov/nchs/nhanes/nhanes_questionnaires.htm. (Accessed 2 December 2015)

Crimmins, E.M., Kim, J.K., Seeman, T.E., 2009. Poverty and biological risk: the earlier "Aging" of the poor. J. Gerontol. Biol. 64 (2), 286-292. doi:10.1093/gerona/ gln010.

de Weerth, C., Buitelaar, J.K., 2005. Physiological stress reactivity in human pregnancy-a review. Neurosci. Biobehav. Rev. 29 (2), 295-312. doi:10.1016/j. neubiorev.2004.10.005.

Felitti, V.J., Anda, R.F., Nordenberg, D., Williamson, D.F., Spitz, A.M., Edwards, V., .... Marks, J.S., 1998. Relationship of childhood abuse and household dysfunction to many of the leading causes of death in adults - The adverse childhood experiences (ACE) study. Am. J. Prev. Med. 14 (4), 245-258. doi:10.1016/ S0749-3797(98)00017-8.

Gilbert, R., Widom, C.S., Browne, K., Fergusson, D., Webb, E., Janson, S., 2009. Child maltreatment 1: burden and consequences of child maltreatment in high-income countries. Lancet 373 (9657), 68-81. doi:10.1016/S0140-6736(08) 61706-7.

Hux, V.J., Roberts, J.M., 2015. A potential role for allostatic load in preeclampsia. Matern. Child Health J. 19 (3), 591-597. doi:10.1007/s10995-014-1543-7.

Juster, R.P., McEwen, B.S., Lupien, S.J., 2010. Allostatic load biomarkers of chronic stress and impact on health and cognition. Neurosci. Biobehav. Rev. 35 (1), 216. doi:10.1016/j.neubiorev.2009.10.002.

Li, Y., Rosemberg, M.S., Dalton, V.K., Lee, S.J., Seng, J.S., 2019. Exploring the optimal allostatic load scoring method in women of reproductive age. J. Adv. Nurs. doi:10.1111/jan.1401410.1111/jan.14014.

McEwen, B.S., 2006. Protective and damaging effects of stress mediators: central role of the brain. Dialogues Clin. Neurosci. 8 (4), 367-381.

McEwen, B.S., Stellar, E., 1993. Stress and the individual. Mechanisms leading to disease. Arch. Intern. Med. 153 (18), 2093-2101.

Morrison, S., Shenassa, E.D., Mendola, P., Wu, T., Schoendorf, K., 2013. Allostatic load may not be associated with chronic stress in pregnant women, Nhanes 19992006. Ann. Epidemiol. 23 (5), 294-297. doi:10.1016/j.annepidem.2013.03.006.

Nandi, A., Glymour, M.M., Subramanian, S.V., 2014. Association among socioeconomic status, health behaviors, and all-cause mortality in the United States. Epidemiology 25 (2), 170-177. doi:10.1097/EDE.0000000000000038.

R Core Team, 2017. R: A language and Environment For Statistical Computing. R Foundation for Statistical Computing, Vienna, Austria https://www.R-project. org/.

Rubin, D.B., 2004. Multiple Imputation For Nonresponse in Surveys. John Wiley \& Sons.

Seeman, T., Glei, D., Goldman, N., Weinstein, M., Singer, B., Lin, Y.H., 2004. Social relationships and allostatic load in Taiwanese elderly and near elderly. Soc. Sci. Med. 59 (11), 2245-2257. doi:10.1016/j.socscimed.2004.03.027.

Soma-Pillay, P., Nelson-Piercy, C., Tolppanen, H., Mebazaa, A., 2016. Physiological changes in pregnancy. Cardiovasc. J. Afr. 27 (2), 89-94. doi:10.5830/ CVJA-2016-021.

von Versen-Hoeynck, F.M., Hubel, C.A., Gallaher, M.J., Gammill, H.S., Powers, R.W. 2009. Plasma levels of inflammatory markers neopterin, sialic acid, and Creactive protein in pregnancy and preeclampsia. Am. J. Hypertens. 22 (6), 687692. doi:10.1038/ajh.2009.54.

Wallace, M.E., Harville, E.W., 2013. Allostatic load and birth outcomes among white and black women in New Orleans. Matern. Child Health J. 17 (6), 1025-1029. doi:10.1007/s10995-012-1083-y.

Watts, D.H., Krohn, M.A., Wener, M.H., Eschenbach, D.A., 1991. C-reactive protein in normal pregnancy. Obstet. Gynecol. 77 (2), 176-180. http://www.ncbi.nlm.nih. gov/pubmed/1988876.

Yuan, Y.C., 2010. Multiple Imputation For Missing Data: Concepts and New Development (Version 9.0). SAS Institute Inc, Rockville, MD, p. 49. 